\title{
Comparative Assessment of Adherence Measures and Resource Use in SSRI/SNRI-Treated Patients with Depression Using Second-Generation Antipsychotics or L-Methylfolate as Adjunctive Therapy
}

Rolin L. Wade, RPh, MS; Sylvia L. Kindermann, MPH; Qingjiang Hou, MS; and Michael E. Thase, MD

\begin{abstract}
BACKGROUND: Antidepressant monotherapy is effective in achieving treatment remission in only approximately one third of patients with depression, and even switching to a second antidepressant brings the cumulative remission rate to only $50 \%-55 \%$. This has led to an interest in augmentation therapy for the management of treatment-resistant depression.
\end{abstract}

OBJECTIVES: To assess (a) selective serotonin reuptake inhibitor/selective norepinephrine reuptake inhibitor (SSRI/SNRI) adherence when augmented with second-generation atypical antipsychotics (SGAs) or L-methylfolate using a modified application of the Healthcare Effectiveness Data and Information Set (HEDIS) acute medication management (AMM) measures at the time of augmentation, and (b) the depression-specific and total health care cost, comparing the 2 forms of augmentation therapy in the treatment of depressive disorder.

METHODS: Patients with a diagnosis of depression and a pharmacy claim for an SSRI/SNRI between January 1, 2006, and December 31, 2009 (index date), and receiving concomitant augmentation therapy with either an SGA or L-methylfolate (augmentation date), were identified in the MarketScan database and followed for $\mathbf{2 3 1}$ days (follow-up). Patients were excluded for having any pharmacy claim for an antidepressant or SGA 90 days pre-index; having an L-methylfolate claim 6 months pre-index; age $<18$ years on the index date; or a diagnosis of pregnancy, dementia, psychotic-related mental disorders, Alzheimer's disease, or Parkinson's disease in the 12-month preindex period. Propensity score matching (3:1 ratio, atypical antipsychotic to L-methylfolate) was used to select the final study cohorts, using covariates of age, gender, comorbidities, index SSRI/SNRI, and index SSRI/SNRI dose. Adherence to antidepressant therapy was measured from the augmentation date and included a modified application of the HEDIS (mHEDIS) AMM acute and chronic phase measures as well as the 6-month medication possession ratio. Health care utilization and cost were measured for the 6-month postaugmentation period and included both total as well as depressionrelated utilization/cost. Comparisons between the closely matched SGA and L-methylfolate-augmented cohorts were made using chi-square tests for binary measures and t-tests for continuous measures.

RESULTS: Following propensity score matching, 4,053 SGA and 1,351 L-methylfolate patients were found to have augmentation of the index SSRI/ SNRI within 12 months of the index date. The comparison groups were well matched on age, gender, comorbidities, and the type and dose of the SSRI/ SNRI being augmented. The most common antidepressants augmented in both groups were escitalopram, duloxetine, and venlafaxine. Mean (standard deviation [SD]) time from index to augmentation was 73.5 [96.7] days for SGA and 105.9 [108.7] days for L-methylfolate $(P<0.001)$. The most common SGAs utilized for augmentation were quetiapine, aripiprazole, and risperidone. L-methylfolate was primarily dosed at $7.5 \mathrm{mg} /$ day. The MHEDIS AMM acute phase measure was met by $68.7 \%$ of the SGA cohort and $78.7 \%$ of the L-methylfolate cohort $(P<0.001)$. The mHEDIS continuation phase measure was met by $50.3 \%$ of the SGA cohort and $62.1 \%$ of the L-methylfolate cohort $(P<0.001)$ following augmentation. Medical utilization (inpatient, emergency department, and outpatient) was significantly higher for the SGA group, while total prescription utilization was significantly higher in the L-methylfolate group. Mean [SD] total 6-month postaugmentation costs for the SGA group was $\$ 8,499$ [\$13,585] and $\$ 7,371$ [ $\$ 12,404]$ for the L-methylfolate group $(P=0.005)$, and 6-month depression-related costs were $\$ 2,688$ [\$4,201] for the SGA group and $\$ 1,613$ [ $\$ 2,315]$ for the L-methylfolate group $(P<0.001)$.

CONCLUSIONS: Patients who augmented SSRI/SNRI therapy with SGA or L-methylfolate achieved mHEDIS AMM acute phase and continuation phase adherence scores of $69 \%-79 \%$ and $50 \%-62 \%$, respectively. These modified scores exceeded the 2012 national median benchmarks for unmodified HEDIS AMM measures for commercial health plans. In this study, augmentation with L-methylfolate was associated with significantly higher adherence measures compared with augmentation with SGA. In addition, health care utilization and total health care costs, as well as depression-related costs, were significantly lower in the L-methylfolate augmentation group compared with augmentation with SGA.

J Manag Care Pharm. 2014;20(1):76-85

Copyright $\odot 2014$, Academy of Managed Care Pharmacy. All rights reserved.

\section{What is already known about this subject}

- A large percentage of patients with depressive disorder fail to achieve remission using antidepressant monotherapy even after switching antidepressant medication. The STAR*D trial failed to demonstrate the superiority of any individual antidepressant option and reported a remission rate of only 50\%-55\% following a second-step treatment attempt. This has led to an interest in augmentation of antidepressant therapy, in which evidence is building that augmentation may be an effective strategy in treatment-resistant depression. 


\section{Comparative Assessment of Adherence Measures and Resource Use in SSRI/SNRI-Treated Patients with Depression Using Second-Generation Antipsychotics or L-Methylfolate as Adjunctive Therapy}

\section{What is already known about this subject (continued)}

- The effectiveness of second-generation atypical antipsychotics (SGA) augmentation in depression has been well studied, and several agents now carry approved labeling for such use. There is a neurophysiological rationale for folate augmentation of antidepressant therapy involving both biochemical and genetic factors, and this strategy has been studied for more than 30 years. Recent clinical trial evidence suggests that L-methylfolate, which is FDA-labeled as a prescription medical food and is available as a pharmacy benefit in some health plans, may be effective as an adjunctive therapy for treatment-resistant depression.

- Despite evidence of effectiveness, little is known about antidepressant adherence rates or costs in patient populations utilizing SGA or L-methylfolate augmentation therapy.

\section{What this study adds}

- Patients who augmented selective serotonin reuptake inhibitor/ selective norepinephrine reuptake inhibitor therapy with SGA or L-methylfolate achieved antidepressant medication management (AMM) acute phase and continuation phase adherence scores of 69\%-79\% and 50\%-62\%, respectively, using a modified National Committee for Quality Assurance's Healthcare Effectiveness Data and Information Set (mHEDIS) AMM score. The modified scores represent AMM HEDIS measures scored following augmentation rather than being scored upon antidepressant initiation. These modified scores exceeded the 2012 national median benchmarks for unmodified HEDIS AMM measures for commercial health plans.

- Adherence scores following L-methylfolate augmentation were significantly higher than those for SGAs.

- Patients receiving augmentation of antidepressant therapy with L-methylfolate demonstrated lower health care utilization, lower all-cause costs, and lower depression-related costs than patients augmenting with SGA for depression.

$\mathrm{M}$ ajor depressive disorder (MDD) is a common, highly recurrent, and potentially fatal illness and has a lifetime prevalence in the United States of approximately $16 \%{ }^{1}$ It is estimated that the societal costs of depression, including those resulting from lost earnings, premature death, and treatment costs, translate into a economic burden of more than $\$ 83$ billion. ${ }^{2}$ Patients with MDD have increased rates of morbidity and mortality, functional impairment, reduced quality of life, and increased risk of suicide. ${ }^{3}$ Studies have shown that despite a wide range of options for treating MDD, up to $40 \%$ of patients fail to respond to treatment, even after fourth-line therapy, based on a validated self-report scale. ${ }^{4}$ Patients who do not respond to antidepressants consume a disproportionally larger share of health care resources and have lower work productivity than patients that respond to treat- ment. $^{2}$ As even sequential monotherapy with antidepressants fails to produce an adequate response to treatment for a large proportion of patients, augmentation of antidepressant therapy with a second antidepressant, atypical antipsychotics, lithium salts, or other agents has been suggested as a solution when traditional therapy fails. ${ }^{5,6}$ Evidence is accumulating showing that combination therapy in treatment of MDD may produce higher remission rates and lower relapse rates than traditional monotherapy, ${ }^{6}$ either as an initial treatment plan or as a strategy for nonresponse to initial treatment. ${ }^{7}$ However, many of the commonly used augmentation strategies are associated with an increase in cost burden as well as higher rates of adverse events, both of which may lead to poor adherence to treatment.

The use of antipsychotics to augment antidepressants for the treatment of depression has been reported since the 1960s. ${ }^{8}$ Conventional antipsychotics have largely been replaced by second-generation antipsychotics (SGAs), perhaps primarily because the newer drugs are associated with fewer extrapyramidal side effects, such as restlessness and repetitive involuntary muscle movements, and have a lower risk for causing tardive dyskinesia than the older agents. Meta-analyses and both the 2006 and 2011 Agency for Healthcare Research and Quality reviews of the efficacy of SGAs confirmed that at least 4 of the SGAs were efficacious when added to selective serotonin reuptake inhibitors/selective norepinephrine reuptake inhibitors (SSRIs/SNRIs) in studies of patients with MDD. ${ }^{9-11}$ Moreover, 3 SGAs (aripiprazole, quetiapine, and olanzapine specifically in combination with fluoxetine) have been approved by the U.S. Food and Drug Administration for this indication. While effective, adjunctive therapy with SGAs has some limitations and disadvantages, including the high cost of many agents and the risks of weight gain and other metabolic complications (e.g., dyslipidemia, hypertriglyceridemia, glucose dysregulation, diabetes mellitus, and hyperprolactinemia), as well as less common risks such as tardive dyskinesia, neuroleptic malignant syndrome, and QTc prolongation., ${ }^{7,12,13}$

In contrast, folate and related compounds, whose role in depression has been recognized for over 20 years, are not associated with the metabolic complications observed with SGAs. ${ }^{13-17}$ The folate cycle plays a central role in the production of catecholamine neurotransmitters, and increasing the synaptic concentration of these transmitters are believed to be one mechanism of action for many antidepressants. ${ }^{18}$ Rather than blocking catecholamine reuptake as with the SSRI/SNRI class of compounds, folate works at the presynaptic level to support catecholamine production. ${ }^{17}$ A meta-analysis of 11 studies with more than 15,000 participants found a significant relationship between low folate status and depression (pooled adjusted odds ratio 1.42, 95\% confidence interval 1.10-1.83). ${ }^{19}$ Folic acid must undergo enzymatic reduction by methyltetrahydrofolate reductase (MTHFR) to become biologically active. ${ }^{20}$ The heterozygous polymorphism of MTHFR has been found in $47 \%$ 


\section{Comparative Assessment of Adherence Measures and Resource Use in SSRI/SNRI-Treated Patients with Depression Using Second-Generation Antipsychotics or L-Methylfolate as Adjunctive Therapy}

of the normal population and the homozygous polymorphism in $11 \%$ of the population. These polymorphisms are known to cause a reduction in MTHFR activity of 34\% and $71 \%$, respectively. ${ }^{19,21,22}$ Folate deficiency can also be caused by drugs (such as anticonvulsants, antibiotics, and oral contraceptives), malabsorption syndromes, chronic diseases, and alcohol use. ${ }^{23}$ Depression is often associated with weight fluctuation, including anorexia and weight loss, which suggests that low folate levels could be both a cause and a consequence of depression. ${ }^{24}$ Low folate blood levels have been associated with poorer or slower response to fluoxetine for $\mathrm{MDD}^{25,26}$ and higher folate levels have been associated with better response to antidepressants. ${ }^{27}$ Folate has been studied as an adjunctive treatment with fluoxetine, with significantly greater improvement in those receiving folate especially among female patients. ${ }^{28}$ L-methylfolate is the primary biologically active isomer of folate, does not require MTHFR for biological activity, and is the form of folate that is transported across the blood-brain barrier. The 2010 American Psychiatric Association guidelines state, "Considering the modest evidence that supports folate as an augmentation strategy and its attractive risk-benefit profile, folate can be recommended as a reasonable adjunctive strategy for major depressive disorder that carries little risk."'

From a regulatory perspective, L-methylfolate is classified as a medical food that differs from dietary supplements in that medical foods are prescribed and are intended for dietary management of a disease or condition for which nutritional requirements are established. The compound is only available as a prescription product in the United States and is indicated for the distinct nutritional requirements of individuals who have suboptimal L-methylfolate levels in the cerebrospinal fluid, plasma, and/or red blood cells and have MDD, with particular emphasis as adjunctive support for individuals who are on an antidepressant. ${ }^{29}$ While some U.S. payers do not include medical foods as a covered benefit, those that do cover them as a pharmacy benefit in a manner similar to other prescription products. Several studies have demonstrated greater efficacy of SSRI/SNRI therapy in MDD when combined with L-methylfolate, providing a more rapid improvement in symptoms and higher remission rates. ${ }^{15,16,30}$ A recent doubleblind, placebo-controlled trial using a sequential parallel group design demonstrated the clinical effectiveness of L-methylfolate in patients with SSRI-resistant depression, with a significant difference in both response rate and degree of improvement in the L-methylfolate group compared with placebo. ${ }^{31}$

Another important factor that moderates the effectiveness of treatments for MDD is the likelihood of adherence to therapy. The importance of medication adherence is mentioned often in the American Psychiatric Association guidelines, ${ }^{7}$ and the National Committee for Quality Assurance (NCQA) Healthcare Effectiveness Data and Information Set (HEDIS) Antidepressant Medication Management (AMM) measures utilize adherence to antidepressant therapy as a key measure. ${ }^{32}$ There is evidence that health care resources and costs are lower in patients who maintain continuous therapy with antidepressants compared with those who discontinue early, ${ }^{33,34}$ although not all studies are in agreement, as an administrative claims study found that costs were higher in NCQA guideline-adherent patients in the 6 months following initiation of therapy. ${ }^{35}$ There is very little published information regarding the impact of augmentation therapy on adherence to antidepressant medication or the impact augmentation may have on health care resource utilization. With the increased interest in adjuncts to SSRI/ SNRI therapy in MDD, in particular with increasing SGA use and recent clinical trials examining L-methylfolate, we sought to examine the adherence rates, using a modified application of the NCQA HEDIS AMM measures, and 6-month health care resource costs in patients with MDD augmenting SSRI/ SNRI therapy with 1 of these 2 augmentation strategies. We hypothesized that adjunctive therapy with both SGAs and L-methylfolate would result in acute phase and continuation phase adherence rates that exceed the 2012 median HEDIS benchmarks and thresholds. We also hypothesized that we would observe differences in the 6-month health care resource utilization between the 2 augmentation strategies, predicting that the lower ingredient cost during the study period, as well as the lower side effect burden of L-methylfolate (in contrast to SGAs), would result in lower overall utilization of health care resources.

This study describes the demographic, clinical, and treatment characteristics of patients with depression who first initiated therapy with an SSRI/SNRI and subsequently were prescribed adjunctive therapy with either an SGA or L-methylfolate. Since our dataset does not include validated indices of symptomatic outcome, our primary interest was in assessing antidepressant adherence outcomes using a modified application of the NCQA AMM measures, and the secondary objective was to assess resource utilization and costs across the 2 populations.

\section{Methods}

This retrospective cohort study utilized the Truven Health Analytics MarketScan Commercial Claims and Encounter and the Medicare Supplemental databases, which capture patientlevel demographic and clinical characteristics, medical and pharmacy utilization, and expenditures across a large U.S. population. The study data extract period was January 1, 2006, to December 31, 2010. The intake patient identification period was January 1, 2007, to December 31, 2009, thereby allowing 12 -month pre- and post-index periods to capture pre-existing comorbidities, outcomes of interest, and exclusionary criteria.

Patients were included in the study if they had a pharmacy claim for an SSRI or SNRI, as well as at least 1 medical claim indicating a clinical visit coded for depression (International 


\section{Comparative Assessment of Adherence Measures and Resource Use in SSRI/SNRI-Treated Patients with Depression Using Second-Generation Antipsychotics or L-Methylfolate as Adjunctive Therapy}

\section{TABLE 1 Demographics: Pre- and Post-Propensity Score Matching}

\begin{tabular}{|c|c|c|c|c|c|c|c|c|c|}
\hline \multirow{4}{*}{$\begin{array}{l}\text { Age at index, years } \\
\text { Gender }\end{array}$} & \multicolumn{4}{|c|}{ Prior to PS Matching } & \multicolumn{4}{|c|}{ PS Matched Groups } & \multirow[b]{2}{*}{$P$ Value $^{\mathrm{a}}$} \\
\hline & \multicolumn{2}{|c|}{$\begin{array}{c}\text { LM } \\
\mathrm{n}=1,351\end{array}$} & \multicolumn{2}{|c|}{$\begin{array}{c}\text { SGA } \\
\mathrm{n}=11,161\end{array}$} & \multicolumn{2}{|c|}{$\begin{array}{c}\text { LM } \\
\mathrm{n}=1,351\end{array}$} & \multicolumn{2}{|c|}{$\begin{array}{c}\text { SGA } \\
\mathbf{n}=4,053\end{array}$} & \\
\hline & \multicolumn{2}{|c|}{$\begin{array}{c}\text { Mean [SD] } \\
45.63[12.91]\end{array}$} & \multicolumn{2}{|c|}{$\begin{array}{c}\text { Mean [SD] } \\
43.78[15.80]\end{array}$} & \multicolumn{2}{|c|}{$\begin{array}{c}\text { Mean [SD] } \\
45.63[12.91]\end{array}$} & \multicolumn{2}{|c|}{$\begin{array}{c}\text { Mean [SD] } \\
45.37[13.98]\end{array}$} & 0.553 \\
\hline & $\mathbf{n}$ & $\%$ & $\mathbf{n}$ & $\%$ & $\mathrm{n}$ & $\%$ & $\mathrm{n}$ & $\%$ & \\
\hline Male & 332 & 24.6 & 4,594 & 41.2 & 332 & 24.6 & 1,045 & 25.8 & 0.377 \\
\hline Female & 1,019 & 75.4 & 6,567 & 58.8 & 1,019 & 75.4 & 3,008 & 74.2 & \\
\hline Pre-index comorbidities & $\mathrm{n}$ & $\%$ & $\mathrm{n}$ & $\%$ & $\mathbf{n}$ & $\%$ & $\mathrm{n}$ & $\%$ & \\
\hline Cancer (solid tumor excluding skin other than melanoma) & 1 & 0.1 & 68 & 0.6 & 1 & 0.1 & 4 & 0.1 & 0.796 \\
\hline Hematologic malignancy & 1 & 0.1 & 13 & 0.1 & 1 & 0.1 & 2 & 0.1 & 0.739 \\
\hline Diabetes, any & 13 & 1.0 & 270 & 2.3 & 13 & 1.0 & 36 & 0.9 & 0.804 \\
\hline Dyslipidemia & 22 & 1.6 & 346 & 3.1 & 22 & 1.6 & 58 & 1.4 & 0.603 \\
\hline Obesity & 11 & 0.8 & 195 & 1.8 & 11 & 0.8 & 33 & 0.8 & 1.000 \\
\hline Hypertension & 35 & 2.6 & 909 & 8.1 & 35 & 2.6 & 96 & 2.4 & 0.646 \\
\hline Coronary and peripheral artery disease & 12 & 0.9 & 263 & 2.4 & 12 & 0.9 & 36 & 0.9 & 1.000 \\
\hline Cerebrovascular disease & 9 & 0.7 & 195 & 1.8 & 9 & 0.7 & 30 & 0.7 & 0.781 \\
\hline Heart failure & 12 & 0.9 & 182 & 1.6 & 12 & 0.9 & 36 & 0.9 & 1.000 \\
\hline Atrial fibrillation & 12 & 0.9 & 225 & 2.0 & 12 & 0.9 & 32 & 0.8 & 0.727 \\
\hline Asthma & 11 & 0.8 & 226 & 2.0 & 11 & 0.8 & 24 & 0.6 & 0.378 \\
\hline Chronic obstructive pulmonary disease/bronchiectasis & 0 & 0.0 & 145 & 1.3 & 0 & 0.0 & 16 & 0.4 & 0.021 \\
\hline Chronic liver disease and cirrhosis & 2 & 0.2 & 56 & 0.5 & 2 & 0.2 & 4 & 0.1 & 0.637 \\
\hline Peptic/upper GI ulcer & 1 & 0.1 & 23 & 0.2 & 1 & 0.1 & 2 & 0.1 & 0.739 \\
\hline Inflammatory bowel disease & 3 & 0.2 & 44 & 0.4 & 3 & 0.2 & 12 & 0.3 & 0.654 \\
\hline Chronic kidney disease & 1 & 0.1 & 61 & 0.6 & 1 & 0.1 & 1 & 0.0 & 0.414 \\
\hline ESRD & 0 & 0.0 & 8 & 0.1 & 0 & 0.0 & 0 & 0.0 & 1.000 \\
\hline Dialysis & 0 & 0.0 & 17 & 0.2 & 0 & 0.0 & 2 & 0.1 & 0.414 \\
\hline HIV/AIDS & 0 & 0.0 & 17 & 0.2 & 0 & 0.0 & 0 & 0.0 & 1.000 \\
\hline Arthritic conditions & 9 & 0.7 & 201 & 1.8 & 9 & 0.7 & 24 & 0.6 & 0.762 \\
\hline Fibromyalgia & 5 & 0.4 & 55 & 0.5 & 5 & 0.4 & 11 & 0.3 & 0.563 \\
\hline Chronic fatigue & 2 & 0.2 & 10 & 0.1 & 2 & 0.2 & 0 & 0.0 & 0.014 \\
\hline Episodic mood disorders (bipolar disorders) & 11 & 0.8 & 773 & 6.9 & 11 & 0.8 & 26 & 0.6 & 0.505 \\
\hline Anxiety disorders & 39 & 2.9 & 968 & 8.7 & 39 & 2.9 & 105 & 2.6 & 0.558 \\
\hline Alcohol or drug abuse & 45 & 3.3 & 1,216 & 10.9 & 45 & 3.3 & 114 & 2.8 & 0.329 \\
\hline Insomnia disorders & 8 & 0.6 & 154 & 1.4 & 8 & 0.6 & 29 & 0.7 & 0.634 \\
\hline
\end{tabular}

aPS matched groups.

ESRD = end stage renal disease; $G I=$ gastrointestinal; HIV/AIDS = human immunodeficiency virus/acquired immunodeficiency syndrome; LM =L-methylfolate; PS = propensity score; $S D=$ standard deviation; $S G A=$ second-generation antipsychotic.

Classification of Diseases, Ninth Revision, Clinical Modification [ICD-9-CM] codes 296.2x, 296.3x, 300.4, 309.1, 311) in either the primary or secondary position within the intake period. The patients' first SSRI/SNRI claim during the identification intake period served as their study index date. Due to the observed differences in dispensed days' supply for the various study agents (SGAs are commonly dispensed in a 30-day supply and L-methylfolate in a 100-day supply), to serve as a washout period, patients with 1 or more prescription claims for an antidepressant of any type or for an SGA in the 90 days prior to the index date, or with 1 or more prescription claims for L-methylfolate in the 6 months preceding the index date, were excluded from the analysis. Additional exclusions were pregnancy 12 months pre- or post-index; lack of continu- ous health plan enrollment 12 months prior to and following the index date; age $<18$ years on the index date; or a primary diagnosis of dementia, psychotic-related mental disorders, Alzheimer's disease, or Parkinson's disease (ICD-9-CM codes 290.xx, 295.xx, 297.xx, 298.xx, 331.x, 332.x, 345.xx) in the 12 -month pre-index period. Finally, patients required augmentation with either an SGA (aripiprazole, clozapine, olanzapine, paliperidone, quetiapine, risperidone, and ziprasidone) or L-methylfolate within 12 months of the index date, as well as at least 231 days of continuous eligibility following the augmentation date. Patients receiving augmentation with both SGA and L-methylfolate in the 12-month post-index period were excluded. 


\section{Comparative Assessment of Adherence Measures and Resource Use in SSRI/SNRI-Treated Patients with Depression Using Second-Generation Antipsychotics or L-Methylfolate as Adjunctive Therapy}

\section{FIGURE 1 Selection of Members for the SGA and L-Methylfolate Augmented Comparison Groups}

Members with at least 1 pharmacy claim for an SSRI/SNRI during the intake period January 1, 2007, through December 31, 2009 $\mathrm{n}=1,103,424$

Members with at least 1 medical visit containing a primary or secondary diagnosis of depression during the intake period January 1,2007 through December 31, 2009

$n=250,715$

Members after exclusions due to pre-index utilization of antidepressants, second-generation antipsychotics, L-methylfolate; diagnosis of pregnancy, dementia, psychosis, or Parkinson's; less than age 18 on index date; or with at least 12 months pre-index and 231 days post-index continous eligibility $\mathrm{n}=220,827$

Members with augmentation with SGA or L-methylfolate within 1 year of the index date and within 1.25x days' supply of an SSRI/SNRI $n=12,763$

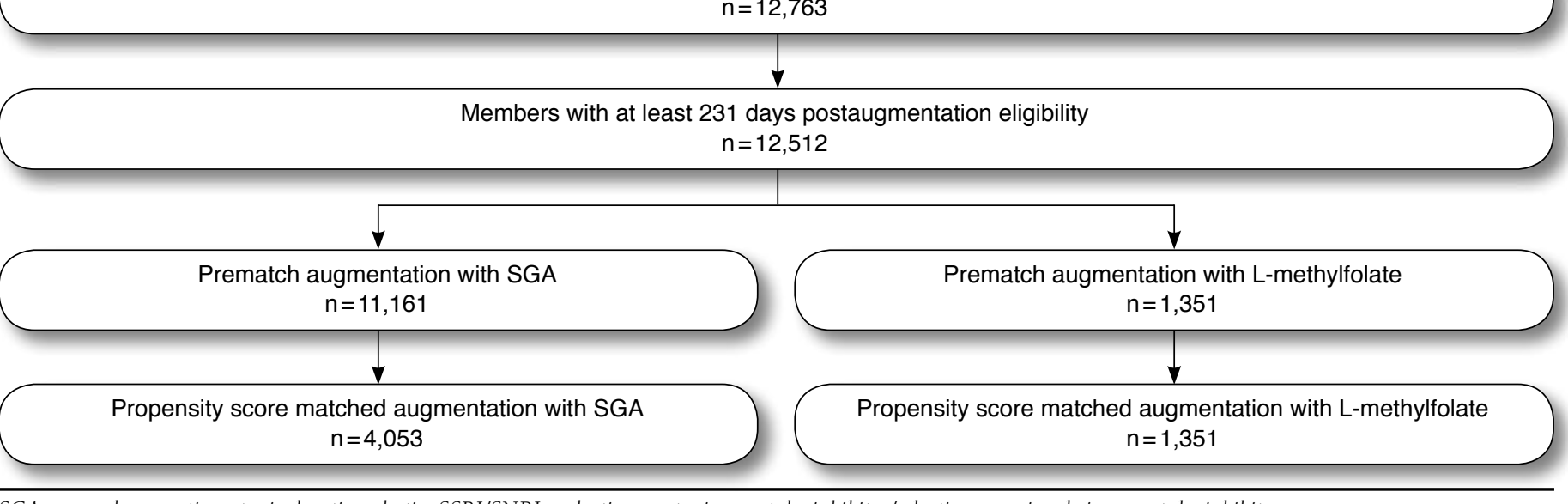

SGA = second-generation atypical antipsychotic; SSRI/SNRI=selective serotonin reuptake inhibitor/selective norepinephrine reuptake inhibitor

Augmentation with an SGA or L-methylfolate was defined as at least 1 prescription claim for either agent, the first claim occurring within 12 months following the index date and within $1.25 \mathrm{x}$ the days' supply period of an SSRI/SNRI claim (either index date claim or a subsequent claim), supporting that adjunctive therapy overlapped with SSRI/SNRI therapy, while allowing for an adherence rate for the SSRI/SNRI of between 80\%-100\%. The date of the first claim for the SSRI/SNRI agent used in combination with an SGA or L-methylfolate is the augmentation date.

Propensity score (PS) matching was utilized for selection of the final SGA and L-methylfolate cohorts. A 3:1 (SGA:Lmethylfolate) PS model was adopted using a greedy matching algorithm and nearest neighbor approach. ${ }^{36,37}$ Propensity scores were assigned utilizing covariates of age, gender, and comorbidities (Table 1) where the characteristics had a difference between the L-methylfolate and SGA groups of $P \leq 0.20$. Comorbidities were selected from those commonly observed in populations with depression. ${ }^{38}$ Additional variables forced into the PS model included specific SSRI/SNRI types and the index SSRI/SNRI dose (as defined by prespecified dose cut points, see Appendix [available in online article]). A flowchart of the population selection process is shown in Figure 1.

AMM outcome measures were modified from NCQA definitions and included (a) acute phase, the percentage of eligible members who remained on antidepressant medication continuously for 3 months as determined by at least 84 days' supply of antidepressant drugs during the first 114 days following receipt of the index antidepressant, and (b) continuation phase, the percentage of eligible members who remained on antidepressant medication continuously for 6 months as determined by at least 180 days' supply of antidepressants during the first 231 days following receipt of the augmented antidepressant. It is important to note that the HEDIS measure specifications start with the first antidepressant prescription start date, and 


\section{Comparative Assessment of Adherence Measures and Resource Use in SSRI/SNRI-Treated Patients with Depression Using Second-Generation Antipsychotics or L-Methylfolate as Adjunctive Therapy}

\section{TABLE 2 SSRI/SNRI Utilization Patterns, Propensity Score Matched Groups}

\begin{tabular}{|c|c|c|c|c|c|}
\hline \multirow[b]{2}{*}{ AD (on augmentation date) } & \multicolumn{2}{|c|}{$\mathrm{LM}, \mathrm{n}=1,351$} & \multicolumn{2}{|c|}{$\mathrm{SGA}, \mathrm{n}=4,053$} & \multirow[t]{2}{*}{$P$ Value $^{\mathrm{a}}$} \\
\hline & $\mathrm{n}$ & $\%$ & $\mathrm{n}$ & $\%$ & \\
\hline Desvenlafaxine & 81 & 6.00 & 178 & 4.39 & \multirow{9}{*}{0.052} \\
\hline Duloxetine & 329 & 24.35 & 928 & 22.90 & \\
\hline Citalopram & 69 & 5.11 & 250 & 6.17 & \\
\hline Escitalopram & 313 & 23.17 & 922 & 22.75 & \\
\hline Fluoxetine & 141 & 10.44 & 507 & 12.51 & \\
\hline Venlafaxine & 189 & 13.99 & 615 & 15.17 & \\
\hline Fluvoxamine & 9 & 0.67 & 34 & 0.84 & \\
\hline Paroxetine & 56 & 4.14 & 134 & 3.31 & \\
\hline Sertraline & 164 & 12.14 & 485 & 11.97 & \\
\hline \multicolumn{6}{|l|}{ Dose change in $\mathrm{AD}$, index to augmentation } \\
\hline No change & 1,142 & 84.53 & 3,422 & 84.43 & \multirow{3}{*}{0.931} \\
\hline Higher & 147 & 10.88 & 347 & 8.56 & \\
\hline Lower & 58 & 4.29 & 164 & 4.05 & \\
\hline Patients exceeding AD high-dose cut point (on augmentation date) & 466 & 34.5 & 1,504 & 37.1 & 0.860 \\
\hline AD switch, index date to augmentation & 209 & 15.47 & 631 & 15.57 & 0.965 \\
\hline Time to augmentation date, days, mean [SD] & 105.9 & {$[108.7]$} & 73.5 & {$[96.7]$} & $<0.001^{b}$ \\
\hline \multicolumn{6}{|c|}{$\begin{array}{l}\text { aBy Pearson chi-square test. } \\
\text { bBy } t \text {-test. } \\
\text { AD = antidepressant; } L M=L \text {-methylfolate; SD = standard deviation; SGA=second-generation antipsychotic; SSRI/SNRI=selective serotonin reuptake inhibitor/selective } \\
\text { norepinephrine reuptake inhibitor. }\end{array}$} \\
\hline
\end{tabular}

for purposes of this study, the HEDIS measures were applied to the antidepressant augmentation date, since the outcome of interest was adherence associated with treatment augmentation. Thus, we refer to these measures as a modified application of the HEDIS AMM measures, or mHEDIS. Medication possession ratio (MPR) was calculated as the sum of patients' days' supply/183 days following the SSRI/SNRI augmentation date to provide a continuous measure of adherence for the 6-month period postaugmentation date. ${ }^{39}$ MPR was capped at $100 \%$, and days' supply that extended past the 183-day measurement window was truncated.

All-cause and depression-related utilization and costs were assessed for the 6-month period following the augmentation date. Costs included plan paid amount without member cost share and were adjusted to 2010 dollars using the medical Consumer Price Index. Total utilization and cost estimates included all medical and prescription claims without regard to diagnosis or drug type, while depression-related utilization and costs included only those medical claims with a primary or secondary diagnosis of depression and pharmacy claims for SSRI/SNRI antidepressants, SGAs, and L-methylfolate. Costs were further categorized as inpatient, emergency department, outpatient, or prescription components based on the source of the claim (medical or pharmacy).

Comparisons between the PS-matched SGA and L-methylfolate cohorts were made using descriptive statistics. Differences in the binary and categorical measures such as comorbidities, type of antidepressant, and AMM measures between the groups were evaluated using chi-square tests, while the differences in continuous measures including time to augmentation, MPR, resource use, and cost were evaluated using t-tests. For cost outcomes, a generalized linear model with log link and gamma distribution was used as a sensitivity analysis to test the cost results.

\section{Results}

Of patients receiving SSRI/SNRI therapy, 11,161 received SGA, and 1,351 received L-methylfolate augmentation within 12 months of the index date. The PS match of 3:1 (SGA to L-methylfolate) provided 4,053 SGA and 1,351 L-methylfolateaugmented patients for analysis in the final study cohort. Table 1 presents the pre- and postmatch study group characteristics. PS matching eliminated significant age, gender, and comorbidity differences (with the exception of chronic obstructive pulmonary disease/bronchiectasis) between the cohorts and produced comparison groups that were equivalent with respect to the type and dose of the SSRI/SNRI being augmented (Table 2).

The most commonly augmented antidepressants among the SGA and L-methylfolate groups, respectively, were the following: duloxetine (23\%-24\%), escitalopram (23\%), and venlafaxine (15\%-14\%). In the SGA-augmented group, $37.1 \%$ of the patients exceeded the high-cut point for SSRI/SNRI therapy, while $34.5 \%$ of the L-methylfolate-augmented group exceeded the high-cut point for SSRI/SNRI therapy (Table 2). An equivalent percentage of patients in both groups switched to a different antidepressant from the index date to augmentation, and there were equivalent percentages in both groups that either 


\section{Comparative Assessment of Adherence Measures and Resource Use in SSRI/SNRI-Treated Patients with Depression Using Second-Generation Antipsychotics or L-Methylfolate as Adjunctive Therapy}

\section{TABLE 3 Medication Adherence Outcomes:} Propensity Score Matched Groups

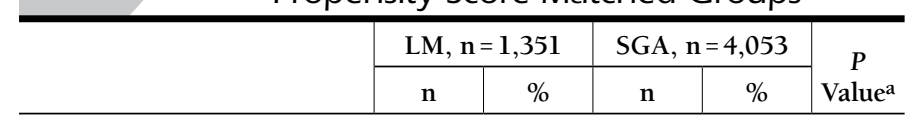

mHEDIS

\begin{tabular}{|l|r|r|r|r|r}
\hline Met acute phase AMM & 1,063 & 78.68 & 2,783 & 68.67 & $<0.001$ \\
\hline
\end{tabular}

\begin{tabular}{l|l|l|l|l|l|l}
\hline Met continuation phase AMM & 839 & 62.10 & 2,040 & 50.33 & $<0.001$ \\
\hline
\end{tabular}
6-month AD therapy MPR

Mean [SD]

Mean [SD]

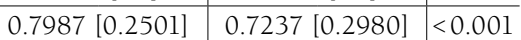

a By Pearson chi-square test.

$A D=$ antidepressant $; A M M=$ Antidepressant Medication Management;

LM = L-methylfolate; $m$ HEDIS = Healthcare Effectiveness Data and Information Set (modified application); $M P R=$ medication possession ratio; $S D=$ standard deviation; $S G A=$ second-generation antipsychotic.

increased or decreased the dose of SSRI/SNRI from index date to augmentation (Table 2).

Small differences ( $5 \%$ or less in absolute terms) were observed in the utilization of other drug therapy in the preaugmentation period when comparing the SGA and L-methylfolate cohorts, including (SGA, L-methylfolate, respectively): bupropion ( $10.0 \%$ vs. $13.6 \%, P=0.0003)$; stimulants $(8.3 \%$ vs. $13.3 \%, P<0.0001)$; thyroid supplementation $(9.7 \%$ vs. $13.3 \%$, $P=0.0002$ ); estrogen and oral contraceptives ( $15.1 \%$ vs. $18.4 \%$, $P=0.0033)$; nonsteroidal anti-inflammatory drugs, including COX-2 (16.0\% vs. 20.4\%, $P=0.0004)$; opioids $(26.8 \%$ vs. $32.8 \%, P<0.0001)$; and beta blockers $(7.8 \%$ vs. $10.4 \%$, $P=0.0046)$.

The most common adjunctive SGAs and their mean daily doses were as follows: quetiapine 39.1\% (109.1 milligrams per day $[\mathrm{mg} / \mathrm{d}])$, aripiprazole $29.5 \%(6.4 \mathrm{mg} / \mathrm{d})$, and risperidone $13.7 \%(1.14 \mathrm{mg} / \mathrm{d})$. The SGA-augmented group had a mean [SD] time to augmentation of 73.5 [96.7] days; $52.0 \%$ augmented in $<30$ days, $32 \%$ in $31-180$ days, and $15.9 \%$ in 181-365 days. The vast majority of patients (96.5\%) prescribed adjunctive L-methylfolate received a dose of $7.5 \mathrm{mg} / \mathrm{day}$. The mean [SD] time to augmentation for the L-methylfolate group was 105.9 [108.7] days, with $36.9 \%$ augmenting in $<30$ days, $37.8 \%$ in $31-180$ days, and $25.2 \%$ in $181-365$ days. There was a significant difference between the time to augmentation for SGA compared with L-methylfolate $(P<0.001)$, indicating that L-methylfolate was typically used later in a treatment episode than SGAs.

Evaluation of the mHEDIS AMM measures following augmentation found that $68.7 \%$ of the SGA-augmented cohort and $78.7 \%$ of the L-methylfolate cohort met the acute phase measures $(P<0.001)$. For the mHEDIS continuation phase measures, $50.3 \%$ of the SGA-augmented cohort and $62.1 \%$ of the L-methylfolate cohort met the measures $(P<0.001)$. In addition, the mean 6-month antidepressant therapy MPR was significantly higher in the L-methylfolate group compared with the SGA-augmented group (Table 3).
Cost and utilization data are presented in Table 4. Allcause 6-month utilization count, including mean inpatient, emergency department, and outpatient visits, were higher in the SGA-augmented cohort compared with those augmented with L-methylfolate. All-cause 6-month mean prescription count was higher in the L-methylfolate cohort compared with the SGA-augmented cohort. Depression-related utilization followed the same pattern, with the exception that 6-month depression-related prescription utilization was equivalent between the 2 groups. Mean all-cause 6-month cost in the SGA-augmented group was approximately $\$ 1,100$ higher than the L-methylfolate group ( $\$ 8,499$ vs. $\$ 7,372, P=0.005)$. The cost difference was reflected in higher 6-month mean costs for inpatient, emergency department, and outpatient visits, as well as prescription costs. Depression-related costs followed the same patterns, with 6-month depression-related costs in the SGA-augmented group being approximately $\$ 1,000$ higher than in the L-methylfolate cohort, reflecting higher costs across all categories $(\$ 2,689$ vs. $\$ 1,613, P<0.001)$. Table 4 also reports significant differences in the component costs for outpatient costs and pharmacy costs for both all-cause and depressionrelated costs. Robust statistical differences within the inpatient and emergency department component costs could not be established due to the excess zero response variables within these components. Multivariate adjustment is commonly applied in these types of studies. In this study, a generalized linear model with log link and gamma distribution was constructed to test the cost results. As the study group matching resulted in closely matched populations, very few variables entered the model and had no influence on the results, with the exception of decreasing the $P$ values for some of the cost differences. We therefore report only the more conservative t-test for the cost outcomes.

\section{Discussion}

The use of adjunctive antidepressant therapies has grown dramatically over the past decade. The principal objective of this study was to compare the impact on SSRI/SNRI adherence and health care utilization between 2 adjunctive strategies, SGA and L-methylfolate, following the initiation of antidepressant monotherapy. In this large administrative claims database, we found that MDD patients were 8 times more likely to receive adjunctive therapy within 1 year with 1 of the SGAs than they were to receive L-methylfolate. We also observed that a large proportion of patients in both groups were augmented within a short time ( $<30$ days) of initiating antidepressant therapy, suggesting that in practice, clinicians are augmenting therapy before establishing the effectiveness of antidepressant monotherapy, as is recommended in practice guidelines such as those published by the American Psychiatric Association. Thus, results from this study indicate that using combination therapy as an initial or early strategy in the treatment of depression 


\section{Comparative Assessment of Adherence Measures and Resource Use in SSRI/SNRI-Treated Patients with Depression Using Second-Generation Antipsychotics or L-Methylfolate as Adjunctive Therapy}

\section{TABLE 4 Costs and Utilization Outcomes: Propensity Score Matched Groups}

\begin{tabular}{|c|c|c|c|c|c|c|c|}
\hline \multirow{3}{*}{$\begin{array}{l}\text { All-Cause Costs } \\
\text { and Utilization } \\
\text { Total costs }\end{array}$} & \multicolumn{3}{|c|}{$\mathrm{LM}, \mathrm{n}=1,351$} & \multicolumn{3}{|c|}{$\mathrm{SGA}, \mathrm{n}=4,053$} & \multirow{3}{*}{$\frac{P \text { Value }^{\mathrm{b}}}{0.005}$} \\
\hline & \multicolumn{2}{|c|}{ Mean $[S D]$} & \multirow{2}{*}{$\begin{array}{l}\text { Median } \\
4,127.22 \\
\end{array}$} & \multicolumn{2}{|c|}{ Mean [SD] } & \multirow{2}{*}{$\begin{array}{l}\text { Median } \\
4,871.88 \\
\end{array}$} & \\
\hline & $7,371.80$ & {$[12,403.60]$} & & $8,499.12$ & {$[13,585.01]$} & & \\
\hline Inpatient component & $1,526.89$ & {$[9,635.56]$} & 0 & $2,067.85$ & {$[10,512.57]$} & 0 & c \\
\hline Outpatient component & $3,265.89$ & {$[5,558.66]$} & $1,638.74$ & $3,586.78$ & {$[5,853.52]$} & $1,808.74$ & 0.029 \\
\hline ED component & 158.03 & [680.03] & 0 & 255.64 & [917.91] & 0 & c \\
\hline Pharmacy component & $2,420.07$ & {$[2,823.33]$} & $1,701.97$ & $2,588.86$ & {$[2,673.62]$} & $1,928.76$ & 0.048 \\
\hline \multicolumn{8}{|l|}{ Visit counts } \\
\hline Inpatient & 0.63 & {$[2.94]$} & 0 & 1.64 & {$[5.85]$} & 0 & c \\
\hline ED & 0.16 & [1.42] & 0 & 0.38 & {$[2.28]$} & 0 & c \\
\hline Outpatient & 16.43 & {$[18.12]$} & 13 & 18.94 & {$[22.15]$} & 15 & $<0.001$ \\
\hline \multicolumn{8}{|c|}{ Depression-Related Costs and Utilization } \\
\hline Total costs & $1,613.26$ & {$[2,314.81]$} & $1,077.7$ & $2,688.77$ & {$[4,201.08]$} & $1,676.51$ & $<0.001$ \\
\hline Inpatient component & 135.99 & {$[1,123.90]$} & 0 & 438.13 & {$[2,618.09]$} & 0 & c \\
\hline Outpatient component & 590.62 & {$[1,230.62]$} & 233.42 & 783.38 & {$[2,214.15]$} & 264.7 & $<0.001$ \\
\hline ED component & 8.80 & [121.79] & 0 & 25.04 & {$[218.77]$} & 0 & c \\
\hline Pharmacy component & 877.85 & {$[816.64]$} & 709.85 & $1,442.22$ & {$[1,412.58]$} & 1,053 & $<0.001$ \\
\hline \multicolumn{8}{|l|}{ Visit counts } \\
\hline Inpatient & 0.22 & [1.43] & 0 & 0.92 & {$[4.33]$} & 0 & c \\
\hline ED & 0.034 & {$[0.487]$} & 0 & 0.1147 & [1.107] & 0 & c \\
\hline Outpatient & 6.03 & {$[10.10]$} & 3 & 6.75 & [12.16] & 3 & 0.03 \\
\hline \multicolumn{8}{|c|}{$\begin{array}{l}\text { aIn } 2010 \text { USD. } \\
b P \text { values are based on t-tests with log transformed response variables, which takes the form of } \log \left[x_{i}+\alpha\right] \text { wh } \\
\text { a small numerical constant that causes the log transformed value to be nonmissing if } x_{i} \text { is zero. } \\
\text { cData contains excess zero response variables and thus are unable to provide a robust log transformed t-test } \\
E D=\text { emergency department; } L M=L \text {-methylfolate; } S D=\text { standard deviation; } S G A=\text { second-generation antipsyc }\end{array}$} \\
\hline
\end{tabular}

may be common in the real-world setting. After equating the groups on relevant demographic and clinical measures using a propensity matching strategy, it was still observed that the patients who were prescribed L-methylfolate were more likely to have received other adjunctive therapies such as thyroid hormone or bupropion. This may explain why L-methylfolate was found to be prescribed later in treatment episodes than the SGAs included in this report. Despite these differences, we found that patients who received adjunctive therapy were above the 2012 median HEDIS benchmarks and thresholds (66\% for acute phase and $53 \%$ for continuation phase $)^{40}$ for adherence to therapy (SGA 69\% and 50\%, L-methylfolate $79 \%$ and $62 \%$, respectively) when measured at the time of augmentation.

With respect to outcomes, the group that received L-methylfolate showed greater adherence to therapy using both the binary mHEDIS AMM as well as the continuous MPR measures when compared with the group that received adjunctive therapy, which was predominately quetiapine, aripiprazole, and risperidone. As the study groups were well matched based on over 20 confounders considered, the causes for adherence differences between the groups is not likely explained by some of the patient-level reasons for nonadherence with medication in patients with depression, such as younger age, substance abuse, cardiovascular morbidity, or use of first-generation agents. ${ }^{41}$ The groups were also well matched on type and dose of SSRI/SNRI, so the differences in adherence are likely caused by the difference in augmentation agents, which include the cost of SGAs at the time of the study and the prevalence and type of adverse events. There is a lack of literature regarding the effect of augmentation on SSRI/SNRI adherence, so these are the first results we are aware of that report on this phenomena. We also observed that patients receiving adjunctive L-methylfolate showed lower overall and depression-specific cost of treatment than did the group receiving an adjunctive SGA. The difference in cost of treatment was not simply attributable to the higher overall prescription costs in the SGA group compared with the L-methylfolate group, since the medical cost categories were also found to be higher in the SGA group for all-cause as well as depression-related costs. The adverse effects of SGAs, such as sedation, sexual dysfunction, glucose abnormalities, and weight gain, which are not associated with L-methylfolate, may have contributed to the additional medical utilization in the SGA group.

It is noteworthy that the majority of patients who received L-methylfolate utilized $7.5 \mathrm{mg} /$ day, a dose that has not been shown to have significant adjunctive efficacy in randomized controlled trials. Since a recent trial has shown that $15 \mathrm{mg} /$ day of L-methylfolate for adjunctive therapy of difficult-to-treat MDD is effective, ${ }^{31}$ it is possible that the findings of the current analysis underestimate the potential utility of this strategy. For 


\section{Comparative Assessment of Adherence Measures and Resource Use in SSRI/SNRI-Treated Patients with Depression Using Second-Generation Antipsychotics or L-Methylfolate as Adjunctive Therapy}

comparison, the doses of aripiprazole, risperidone, and quetiapine were also used at doses much lower than the usual daily dosage of these compounds for antipsychotic therapy. ${ }^{42}$

\section{Limitations}

Several limitations to this study should be noted. First, due to the observational design of the study, associations can be identified, but causality cannot be established. The PS matching design controls for the measured confounders in the 2 study groups, but unmeasured differences between the groups likely remained. In particular, this study was unable to control for disease severity or duration, since such data are unavailable in the database. The SGA group was augmented significantly earlier in the post-index period, an effect that may point to unmeasured confounders, and may have contributed to the higher pharmacy component costs seen in the SGA-augmented group. There were unexplained comorbidity differences between the study groups before matching, with the SGA group generally showing a higher level of comorbidities, which may indicate a treatment selection bias between SGA and L-methylfolate. This may in turn suggest these results may be limited to populations that are similar to the postmatched groups, which following PS matching was quite similar in measured comorbidities. As is true of all retrospective studies where cohort identification is based on the use of ICD-9-CM diagnostic coding, we have to consider the possibility that there was inaccuracy in the coding, but we have no reason to believe there was any systematic bias in diagnostic coding between the study groups.

The generalizability of these results is limited to a commercially insured population and may not be applicable to other populations such as the Medicaid or traditional fee-for-service Medicare populations. We also note that many of the SGAs in this study were available only as branded products during the study period, and while the differences in pharmacy costs between the SGA and L-methylfolate groups may be mitigated as generic versions of SGAs replace the branded versions, the differences in side effects and metabolic effects between SGAs and L-methylfolate will remain. The individual antipsychotic agents may have resulted in different outcomes for each agent, but our study was not designed to examine individual agents, which may be of interest for future study. Finally, the use of specialty psychiatric care was not taken into account, since no information was available as to whether any patient was enrolled in a health plan with a psychiatric carve-out.

\section{Conclusions}

In this study, patients receiving augmentation of antidepressant therapy with L-methylfolate compared with SGA for depression demonstrated lower health care utilization, lower all-cause costs, and lower depression-related costs. Further, patient populations using augmentation therapy with L-methylfolate had significantly higher mHEDIS scores than the SGA group in both acute and continuing phase AMM measures. While further study of the effect of augmentation therapy in MDD is warranted, a benefit design that includes L-methylfolate should be considered among the options available as augmentation therapy to SSRI/SNRI treatment in depression, given the neurochemical basis for activity, recent trials demonstrating clinical effectiveness, and the relatively low cost and adverse event profile.

\section{Authors}

ROLIN L. WADE, RPh, MS, is Principal, Health Economics and Outcomes Research, Real World Evidence Solutions, IMS Health, Parsippany, New Jersey; SYLVIA L. KINDERMANN, MPH, is Scientist, and QINGJIANG HOU, MS, is Scientist/Biostatistician, Cerner Research, Culver City, California; and MICHAEL E. THASE, MD, is Professor of Psychiatry, Perelman School of Medicine, University of Pennsylvania, Philadelphia, Pennsylvania.

AUTHOR CORRESPONDENCE: Rolin Wade, RPh, MS, IMS Health, 11 Waterfront Blvd., Parsippany, NJ 07054. Tel.: 908.236.8972; E-mail: rwade@imshealth.com.

\section{DISCLOSURES}

The work presented in this manuscript was supported by Nestlé Health Science-Pamlab, Inc. Kindermann and Hou are employees of Cerner Research, who contracted with the sponsor to conduct the project. At the time of this study, Wade was an employee of Cerner Research. Thase has been a member of advisory boards and has consulted for Pamlab, Astra Zeneca, Bristol-Myers Squibb, and Eli Lilly and Company.

Study concept and design were contributed by Wade, Kindermann, and Thase, with assistance from Hou. Hou was primarily responsible for data collection, with assistance from Wade. Wade, Kindermann, and Thase were responsible for data interpretation, with assistance from Hou. Kindermann, Wade, and Thase wrote the manuscript, with assistance from Hou, and Lisa Kaspin was primarily responsible for revisions along with Wade and with assistance from Kindermann, Hou, and Thase.

\section{ACKNOWLEDGMENTS}

The authors would like to acknowledge Lisa Kaspin, $\mathrm{PhD}$, for her assistance in editing the final manuscript.

\section{REFERENCES}

1. Kessler RC, Berglund P, Demler O, et al. The epidemiology of major depressive disorder: results from the National Comorbidity Survey Replication (NCS-R). JAMA. 2003;289(23):3095-105

2. Greenberg PE, Kessler RC, Birnbaum HG, et al. The economic burden of depression in the United States: how did it change between 1990 and 2000? J Clin Psychiatry. 2003;64(12):1465-75.

3. Greden F. The burden of disease for treatment-resistant depression. J Clin Psychiatry. 2001; 62(Suppl 16):26-31

4. Rush AJ, Trivedi MH, Wisniewski SR, et al. Acute and longer-term outcomes in depressed outpatients requiring one or several treatment steps: a STAR*D report. Am J Psychiatry. 2006; 163(11):1905-17. 


\section{Comparative Assessment of Adherence Measures and Resource Use in SSRI/SNRI-Treated Patients with Depression Using Second-Generation Antipsychotics or L-Methylfolate as Adjunctive Therapy}

5. Alexopoulos GS. Pharmacotherapy for late-life depression. J Clin Psychiatry. 2011; $72(1): e 04$.

6. Stahl SM. Combining antidepressant therapies from the initiation of treatment: a paradigm shift for major depression. J Clin Psychiatry. 2009; 70(11):1493-94

7. American Psychiatric Association. Practice Guideline for the Treatment of Patients with Major Depressive Disorder. 3rd ed. Arlington, VA: American Psychiatric Association; 2010.

8. Cowen PJ. Revisiting pharmacological management of treatment-resistant depression. Advances in Psychiatric Treatment. 2005;11:19-27.

9. Shekelle P, Maglione M, Bagley S, et al. Comparative effectiveness of offlabel use of atypical antipsychotics. Comparative Effectiveness Review No. 6 (Prepared by the Southern California/RAND Evidence-based Practice Center under Contract No. 290-02-0003.) Rockville, MD: Agency for Healthcare Research and Quality. January 2007. Available at: http://www.ncbi.nlm.nih. gov/books/NBK43235/pdf/TOC.pdf. Accessed November 5, 2013.

10. Maglione M, Ruelaz Maher A, Hu J, et al. Off-label use of atypical antipsychotics: an update. Comparative Effectiveness Review No. 43. (Prepared by the Southern California Evidence-based Practice Center under Contract No. HHSA290-2007-10062-1) Rockville, MD: Agency for Healthcare Research and Quality. September 2011. Available at: http:// www.effectivehealthcare.ahrq.gov/ehc/products/150/778/CER43_OffLabelAntipsychotics_20110928.pdf. Accessed November 5, 2013.

11. Nelson JC, Papakostas GI. Atypical antipsychotic augmentation in major depressive disorder: a meta-analysis of placebo-controlled randomized trials. Am J Psychiatry. 2009;166(9):980-91.

12. Wright BM, Eiland EH, 3rd, Lorenz R. Augmentation with atypical antipsychotics for depression: a review of evidence-based support from the medical literature. Pharmacotherapy. 2013; 33(3):344-59.

13. Newcomer JW. Second-generation (atypical) antipsychotics and metabolic effects: a comprehensive literature review. CNS Drugs. 2005;19 (Suppl 1):S1-S93.

14. Fava M, Mischoulon D. Folate in depression: efficacy, safety, differences in formulations, and clinical issues. J Clin Psychiatry. 2009;70(Suppl 5):S12-S17.

15. Godfrey PS, Toone BK, Carney MW, et al. Enhancement of recovery from psychiatric illness by methylfolate. Lancet. 1990;336(8712):392-95.

16. Procter A. Enhancement of recovery from psychiatric illness by methylfolate. Br J Psychiatry. 1991;159:271-72.

17. Stahl SM. Novel therapeutics for depression: L-methylfolate as a trimonoamine modulator and antidepressant-augmenting agent. CNS Spectr. 2007;12(10):739-44

18. Westenberg HG. Pharmacology of antidepressants: selectivity or multiplicity? J Clin Psychiatry. 1999;60(Suppl 17):S4-S8.

19. Gilbody S, Lightfoot T, Sheldon T. Is low folate a risk factor for depression? A meta-analysis and exploration of heterogeneity. J Epidemiol Community Health. 2007;61(7):631-37.

20. Bottiglieri T. Homocysteine and folate metabolism in depression. Prog Neuropsychopharmacol Biol Psychiatry. 2005;29(7):1103-12.

21. de Bree A, Verschuren WM, Bjorke-Monsen AL, et al. Effect of the methylenetetrahydrofolate reductase $677 \mathrm{C}-->\mathrm{T}$ mutation on the relations among folate intake and plasma folate and homocysteine concentrations in a general population sample. Am J Clin Nutr. 2003;77(3):687-93.

22. Klerk M, Verhoef P, Clarke R, Blom HJ, Kok FJ, Schouten EG. MTHFR 677C-->T polymorphism and risk of coronary heart disease: a meta-analysis JAMA. 2002;288(16):2023-31

23. Alpert JE, Mischoulon D, Nierenberg AA, Fava M. Nutrition and depression: focus on folate. Nutrition. 2000;16(7-8):544-46.

24. Nelson JC. The evolving story of folate in depression and the therapeutic potential of 1-methylfolate. Am J Psychiatry. 2012;169(12):1223-25.
25. Papakostas GI, Petersen T, Mischoulon D, et al. Serum folate, vitamin B12, and homocysteine in major depressive disorder, Part 1: predictors of clinical response in fluoxetine-resistant depression. J Clin Psychiatry. 2004;65(8):1090-95.

26. Papakostas GI, Petersen T, Lebowitz BD, et al. The relationship between serum folate, vitamin B12, and homocysteine levels in major depressive disorder and the timing of improvement with fluoxetine. Int $J$ Neuropsychopharmacol. 2005;8(4):523-28.

27. Alpert M, Silva RR, Pouget ER. Prediction of treatment response in geriatric depression from baseline folate level: interaction with an SSRI or a tricyclic antidepressant. J Clin Psychopharmacol. 2003;23(3):309-13.

28. Coppen A, Bailey J. Enhancement of the antidepressant action of fluoxetine by folic acid: a randomised, placebo controlled trial. J Affect Disord. 2000;60(2):121-30.

29. Deplin package insert. Pamlab LLC. Available at: http://www.deplin. com/request-package-insert/. Accessed December 3, 2013.

30. Fava M, Rush AJ. Current status of augmentation and combination treatments for major depressive disorder: a literature review and a proposal for a novel approach to improve practice. Psychother Psychosom. 2006;75(3):139-53.

31. Papakostas GI, Shelton RC, Zajecka JM, et al. L-Methylfolate as adjunctive trherapy for SSRI-resistant major depression: results of two randomized, double-blind, parallel-sequential trials. Am J Psychiatry. 2012;169(12):1267-74.

32. National Committee for Quality Assurance. The state of health care quality 2006. Available at: http://ipp.jsi.com/Docs/SOHC_2006.pdf. Accessed November 5, 2013

33. Eaddy MT, Druss BG, Sarnes MW, Regan TS, Frankum LE. Relationship of total health care charges to selective serotonin reuptake inhibitor utilization patterns including the length of antidepressant therapy-results from a managed care administrative claims database. J Manag Care Pharm. 2005;11(2):145-50. Available at: http://amcp.org/WorkArea/DownloadAsset. aspx?id=7123.

34. Thompson D, Buesching D, Gregor KJ, Oster G. Patterns of antidepressant use and their relation to costs of care. Am J Manag Care. 1996;2:1239-46.

35. Robinson RL, Long SR, Chang S, et al. Higher costs and therapeutic factors associated with adherence to NCQA HEDIS antidepressant medication management measures: analysis of administrative claims. J Manag Care Pharm. 2006;12(1):43-54. Available at: http://amcp.org/WorkArea/ DownloadAsset.aspx?id=7313.

36. Rosenbaum PR, Rubin DB. The central role of the propensity score in observational studies for causal effects. Biometrika. 1983;70(1):41-55.

37. Rosenbaum PR, Rubin DB. Constructing a control group using multivatiate matched sampling methods that incorporate the propensity score. The American Statician. 1985;39(1):33-38.

38. Caughey GE, Roughead EE, Shakib S, McDermott RA, Vitry AI, Gilbert AL. Comorbidity of chronic disease and potential treatment conflicts in older people dispensed antidepressants. Age Ageing. 2010;39(4):488-94.

39. Hess LM, Raebel MA, Conner DA, Malone DC. Measurement of adherence in pharmacy administrative databases: a proposal for standard definitions and preferred measures. Ann Pharmacother. 2006;40(7-8):1280-88.

40. National Committee for Quality Assurance. Benchmarks and thresholds: 2012 accreditation. August 1, 2012. Available at: http://www.ncqa.org/ tabid/422/Default.aspx. Accessed November 5, 2013.

41. Akincigil A, Bowblis JR, Levin C, Walkup JT, Jan S, Crystal S. Adherence to antidepressant treatment among privately insured patients diagnosed with depression. Med Care. 2007:45(4):363-69.

42. Muench J, Hamer AM. Adverse effects of antipsychotic medications. Am Fam Physician. 2010;81(5):617-22. 
Comparative Assessment of Adherence Measures and Resource Use in SSRI/SNRI-Treated Patients with Depression Using Second-Generation Antipsychotics or L-Methylfolate as Adjunctive Therapy

APPENDIX Dose Range Cut Points for SSRI/SNRI Antidepressants

\begin{tabular}{l|l|l}
\hline SSRI/SNRI & LOW Dose Cut Point & HIGH Dose Cut Point \\
\hline Fluoxetine & $\leq 40 \mathrm{mg} / \mathrm{d}$ & $>40 \mathrm{mg} / \mathrm{d}$ \\
\hline Sertraline & $\leq 100 \mathrm{mg} / \mathrm{d}$ & $>100 \mathrm{mg} / \mathrm{d}$ \\
\hline Paroxetine IR & $\leq 30 \mathrm{mg} / \mathrm{d}$ & $>30 \mathrm{mg} / \mathrm{d}$ \\
\hline Paroxetine ER & $\leq 37.5 \mathrm{mg} / \mathrm{d}$ & $>37.5 \mathrm{mg} / \mathrm{d}$ \\
\hline Fluvoxamine & $\leq 200 \mathrm{mg} / \mathrm{d}$ & $>200 \mathrm{mg} / \mathrm{d}$ \\
\hline Citalopram & $\leq 30 \mathrm{mg} / \mathrm{d}$ & $>30 \mathrm{mg} / \mathrm{d}$ \\
\hline Escitalopram & $\leq 10 \mathrm{mg} / \mathrm{d}$ & $>10 \mathrm{mg} / \mathrm{d}$ \\
\hline Venlafaxine IR & $\leq 150 \mathrm{mg} / \mathrm{d}$ & $>150 \mathrm{mg} / \mathrm{d}$ \\
\hline Venlafaxine ER & $\leq 150 \mathrm{mg} / \mathrm{d}$ & $>150 \mathrm{mg} / \mathrm{d}$ \\
\hline Duloxetine & $\leq 60 \mathrm{mg} / \mathrm{d}$ & $>60 \mathrm{mg} / \mathrm{d}$ \\
\hline Desvenlafaxine & $\leq 50 \mathrm{mg} / \mathrm{d}$ & $>50 \mathrm{mg} / \mathrm{d}$ \\
\hline
\end{tabular}

Note: LOW and HIGH dose cut points were chosen through observation of the dose distribution within the study population. They are not meant to represent dose equivalency among the agents and were used to balance the SSRI/SNRI dose among study groups in propensity score matching.

$\mathrm{mg} / \mathrm{d}=$ milligrams per day; SSRI/SNRI = selective serotonin reuptake inhibitor/ selective norepinephrine reuptake inhibitor. 